\title{
Bond dimension witnesses and the structure of ho- mogeneous matrix product states
}

\author{
Miguel Navascués ${ }^{1,2}$ and Tamás Vértesi ${ }^{3}$ \\ ${ }^{1}$ Department of Physics, Bilkent University, Ankara 06800, Turkey \\ ${ }^{2}$ Institute for Quantum Optics and Quantum Information (IQOQI), Boltzmangasse 3, 1090 Vienna, Austria \\ ${ }^{3}$ Institute for Nuclear Research, Hungarian Academy of Sciences, H-4001 Debrecen, P.O. Box 51, Hungary \\ January 30, 2018
}

For the past twenty years, Matrix Product States (MPS) have been widely used in solid state physics to approximate the ground state of one-dimensional spin chains. In this paper, we study homogeneous MPS (hMPS), or MPS constructed via site-independent tensors and a boundary condition. Exploiting a connection with the theory of matrix algebras, we derive two structural properties shared by all hMPS, namely: a) there exist local operators which annihilate all hMPS of a given bond dimension; and $b$ ) there exist local operators which, when applied over any hMPS of a given bond dimension, decouple (cut) the particles where they act from the spin chain while at the same time join (glue) the two loose ends back again into a hMPS. Armed with these tools, we show how to systematically derive 'bond dimension witnesses', or 2local operators whose expectation value allows us to lower bound the bond dimension of the underlying hMPS. We extend some of these results to the ansatz of Projected Entangled Pairs States (PEPS). As a bonus, we use our insight on the structure of hMPS to: a) derive some theoretical limitations on the use of hMPS and hPEPS for ground state energy computations; b) show how to decrease the complexity and boost the speed of convergence of the semidefinite programming hierarchies described in [Phys. Rev. Lett. 115, 020501 (2015)] for the characterization of finite-dimensional quantum correlations.

\section{Introduction}

The study of condensed matter phases depends crucially on our ability to determine the properties of the ground state of local Hamiltonians defined over a lattice. Not only this problem has been shown to be QMA-hard for general Hamiltonians [1, 17], but already for mesoscopic systems (of $n \sim 40$ particles), even storing the description of a general quantum state in a normal computer becomes an impossible task. This forces condensed matter physicists to resort to quantum state ansatzs in order to understand and study the properties of matter in the low temperature regime.

One ansatz that has proven very useful in this respect is the family of Tensor Network States (TNS) [18], a class of many-body wavefunctions of complexity fixed by a parameter known as bond dimension. In the last few years, TNS have been successfully used to approximate the low energy sector of local Hamiltonians of spin lattices of different dimensions $[5,23,29]$. The ability to approximately compute expectation values in an efficient manner, together with the possibility to conduct optimizations in the thermodynamical limit [25], [6], [21], [13] makes TNS one of the very few avenues to understand the physics of strongly correlated systems.

Because of all the above and further theoretical considerations, in the last years, almost every talk about TNS starts with the speaker reminding the audience that "TNS of low bond dimension are the only physical states of condensed matter systems', or, equivalently, that 'all other rays of the Hilbert space of a many body system are not physically realizable'.

Suppose that we take this last claim at face 
value. That is, we postulate that the laws of Nature are such that the states of condensed matter systems at low temperature are very close to being representable by convex combinations TNS of low bond dimension. This is in effect a physical theory; as such, its limits must be explored to determine to which degree the theory is falsifiable, and thus scientific. Given that all one can hope to estimate in the lab are the expectation values of certain $k$-local observables, what makes TNS special, when compared to any other quantum state? Can we prove that the underlying quantum state cannot possibly be approximated by convex combinations of TNS with low bond dimension, i.e., can we falsify a TNS model? Note that, even if we do not adhere to the "Church of the Low Bond Dimension", we can use the bond dimension of a TNS model as a measure of the complexity of the underlying quantum state. In this sense, an experimental refutation of a TNS model with high bond dimension could be regarded as a benchmark for the quantum control of condensed matter systems.

We lack tools to answer these questions. Note that the naive scheme of lower bounding the bond dimension by estimating the rank of reduced density matrices only works with pure TNS and not convex combinations thereof. Moreover, the physical scenarios which we consider here just allow the experimentalist to estimate averages of two-body reduced density matrices. In addition, the variational methods so commonly used in condensed matter physics to optimize over TNS of fixed bond dimension are useless to refute a TNS model: such a task would require relaxation, rather than variational techniques.

In this paper, we will address these problems for homogeneous Matrix Product States (hMPS) [20], a class of TNS used to model non-critical one-dimensional spin chains with translation invariance.

We start by deriving two surprising features of hMPS: first, for any $D$ we identify local operators which annihilate all hMPS of bond dimension smaller than or equal to $D$. Second, for any $D$ we prove the existence of local operators which, when applied over any hMPS of bond dimension $D$, decouple (cut) the particles where they act from the spin chain while at the same time join (glue) the two loose ends back again into a hMPS.

Armed with these notions, we will define a fam- ily of $k$-local operators with negative eigenvalues whose expectation values are nonetheless positive for all hMPS of a given bond dimension $D$. Each such operator can be used to certify that the quantum state of a non-critical spin chain does not admit an hMPS representation of bond dimension $D$. Moreover, this partial characterization of the dual cone of hMPS allows us to devise general feasibility tests, or automated criteria to falsify hMPS models given limited data about the underlying quantum state, such as a number of experimentally available expectation values.

In addition, we will construct instances of local Hamiltonians of arbitrarily many qubits for which a blind application of hMPS-based optimization methods would fail to estimate the ground state energy. This construction can be generalized to other TNS for optimizations over spin lattices of higher spatial dimensions. We will also exploit the low dimensionality of the space spanned by hMPS and the notion of cut-and-glue operators to decrease the complexity and boost the speed of convergence of the semidefinite programming (SDP) [27] hierarchies described in [15, 16] for the characterization of finite-dimensional quantum correlations.

The structure of this paper is as follows: first, we will introduce local Hamiltonians and MPS, and also a couple of notions from the theory of Matrix Algebras. Then we will reveal a connection between hMPS and polynomials of noncommuting variables, which will allow us to derive non-trivial structural properties of hMPS. Next, we will use these properties to explore the limits of hMPS models and improve the SDP relaxations proposed in $[15,16]$. Finally, we will discuss how some of our results generalize to Projected Entangled Pairs States (PEPS) [23].

Before we proceed, though, a disclaimer is in order: long after the completion of this work, we were made aware that the connection between matrix algebras and MPS had already been pointed out by R. Werner in 2006 [30]. In this encyclopaedia article, Werner also observes that the dimensionality of the space spanned by MPS of a fixed bond dimension is polynomial on the system size. 


\section{Matrix product states and the theory of matrix algebras}

Consider a quantum system composed of $n$ distinguishable particles, each of which has local dimension $d$. A general pure state $|\psi\rangle$ of this ensemble hence lives in $\left(\mathbb{C}^{d}\right)^{\otimes n}$, and so we require $d^{n}$ complex parameters to describe it.

An $n$-site homogeneous Matrix Product State (hMPS) is a state of the form

$|\psi(\omega, A, n)\rangle=\sum_{i_{1}, \ldots, i_{n}=1}^{d} \operatorname{tr}\left(\omega A_{i_{1}} A_{i_{2}} \ldots A_{i_{n}}\right)\left|i_{1}, \ldots, i_{n}\right\rangle$,

where $\omega, A_{1}, \ldots, A_{d}$ are $D \times D$ matrices. Note that in general Matrix Product States (MPS) the matrices $\left\{A_{i}\right\}_{i=1}^{d}$ are taken to be site-dependent [20]. The matrix $\omega$ is a boundary condition, while the parameter $D$ is known as the bond dimension of the state. In order to distinguish it from $d$, the latter is also called the physical dimension.

It can be proven that, for $D$ high enough, all states can be expressed as in eq. (1). However, we will be interested in systems where the value of $D$ does not grow much with the system size $n$. Note that a hMPS of whatever size can be described with $O\left(d D^{2}\right)$ complex parameters. Hence, as long as $D$ is not very big, it pays to use this approximation. Finally, notice that, taking $\omega=\mathbb{I}_{D}$, the state becomes invariant with respect to the permutation $1 \rightarrow 2 \rightarrow \ldots \rightarrow n \rightarrow 1$. Such states are called uniform translation invariant (TI) MPS.

For low values of $D$, computing expectation values of product operators in a hMPS can be carried out in an efficient way. Thus hMPS (in particular, uniform TI MPS) are regularly used to approximate the ground state of $k$-local Hamiltonians of one-dimensional systems, i.e., Hamiltonians of the form:

$$
H=\sum_{j=1}^{n} h_{j},
$$

where $h_{j}$ acts non-trivially on the space of the particles $j, j+1, \ldots, j+k-1$. Given $H$, we will denote by $\langle H\rangle_{D}$ the minimum average value of $H$ achievable with hMPS of bond dimension $D$.

Note that we can always choose $\left\{A_{i}\right\}$ satisfying $\sum_{i=1}^{d} A_{i} A_{i}^{\dagger}=\mathbb{I}_{D}[20]$. This allows us to perform calculations in the thermodynamic limit, i.e., $n \rightarrow \infty$. Indeed, in such a case, the $m$-site reduced density matrix $\rho_{m}$ of the state under consideration is equal to

$\rho_{m}=\sum_{\vec{i}, \vec{j}} \operatorname{tr}\left(A_{j_{m}}^{\dagger} \ldots A_{j_{1}}^{\dagger} \sigma A_{i_{1}} \ldots A_{i_{m}}\right)\left|i_{1}, \ldots, i_{m}\right\rangle\left\langle j_{1}, \ldots j_{m}\right|$

where $\sigma \geq 0$ satisfies $\operatorname{tr}(\sigma)=1$ and $\sum_{i} A_{i}^{\dagger} \sigma A_{i}=$ $\sigma$, and the sum runs over all vectors $\vec{i}, \vec{j} \in$ $\{1, \ldots, d\}^{m}$. With the latter conditions, the above is an infinite MPS (iMPS).

If the state of a finite spin chain can be expressed as a convex combination of hMPS of bond dimension $D$, we will say that it admits a $h M P S$ model of bond dimension $D$. Furthermore, if the TI state of an infinite spin chain can be expressed as a convex combination of iMPS of bond dimension $D$, we will say that it admits an $i M P S$ model.

An important notion in uniform MPS is the concept of injectivity. A uniform $n$-site TI MPS $|\psi\rangle$ with bond dimension $D$ is said to be injective if, for $m \leq n$, the map $\Gamma: B\left(\mathbb{C}^{D}\right) \rightarrow \mathbb{C}^{d^{m}}$ given by $\Gamma(X)=\sum_{i_{1}, \ldots, i_{m}} \operatorname{tr}\left(X A_{i_{1}} \ldots A_{i_{m}}\right)\left|i_{1}, \ldots, i_{n}\right\rangle$ is injective. Equivalently, a uniform TI MPS $|\psi\rangle$ with bond dimension $D$ is not injective if it admits a representation in terms of $D \times D$ blockdiagonal matrices.

We now digress momentarily from the topic of MPS to the theory of matrix algebras. A matrix polynomial identity (MPI) $F(X)$ for dimension $D$ is a polynomial of noncommuting variables $X_{1}, \ldots, X_{d}$ that vanishes when evaluated with matrices of dimension $D$ or lower. For example, any commutator $\left[X_{i}, X_{j}\right]$ is a MPI for $D=1$. A more elaborate example is $\left[\left[X_{1}, X_{2}\right]^{2}, X_{3}\right]$; this polynomial vanishes when evaluated with $2 \times 2$ matrices.

Let us see why: being a commutator, the trace of $Z \equiv\left[X_{1}, X_{2}\right]$ must be zero, and so $Z=\sum_{i=1}^{3} c_{i} \sigma_{i}$, for some complex numbers $c_{1}, c_{2}, c_{3}$ (here $\sigma_{1}, \sigma_{2}, \sigma_{3}$ denote the Pauli matrices). Squaring $Z$ we get $Z^{2}=\left(\sum_{i=1}^{3} c_{i}^{2}\right) \cdot \mathbb{I}$, and thus $\left[Z^{2}, X_{3}\right]=0$ for all $X_{3} \in B\left(\mathbb{C}^{2}\right)$.

MPIs do not only exist for dimensions 1 and 2. In general, it can be proven that any $2 D$-tuple of $D \times D$ matrices $X_{1}, \ldots, X_{2 D}$ must satisfy the standard identity $F_{2 D}(X)=0$ [7], where

$$
F_{N}(X) \equiv \sum_{\pi \in S_{N}} \operatorname{sgn}(\pi) X_{\pi(1)} \ldots X_{\pi(N)} .
$$

Here $S_{N}$ denotes the set of all permutations $\pi$ of $N$ elements. It can also be shown that MPIs for 
matrices of dimension $D$ must necessarily have degree $2 D$ or higher [7].

A concept related to MPIs is that of central matrix polynomials, or polynomials $P(X)$ of noncommuting variables which are proportional to the identity when evaluated with $D \times D$ matrices. E.g.: in $D=1$, any polynomial can be interpreted as a central polynomial. In $D=2$ we already saw an example, namely the polynomial $\left[X_{1}, X_{2}\right]^{2}$. As with MPIs, it can be proven that non-trivial central polynomials (i.e., central polynomials which are not MPIs) exist for all dimensions $D[7]$.

\section{The Physics of MPS}

We will next establish a relation between matrix polynomials and many-body quantum states. From this link, non-trivial structural properties of hMPS will follow almost straightforwardly. Let $X \equiv\left(X_{1}, \ldots, X_{d}\right)$ be any tuple of $d$ noncommuting variables, and let $P(X)$ be a homogeneous polynomial $P\left(X_{1}, \ldots, X_{d}\right)=$ $\sum_{i_{1}, \ldots i_{m}} p_{i_{1}, \ldots, i_{m}} X_{i_{1}} \ldots X_{i_{m}}$ of degree $m$. By $|P(X)\rangle$ we will denote the $m$-particle vector $|P(X)\rangle=\sum_{i_{1}, \ldots i_{m}} p_{i_{1}, \ldots, i_{m}}^{*}\left|i_{1}, \ldots, i_{m}\right\rangle$.

It is immediate that, for any $n$-site hMPS $|\psi\rangle$ of the form (1), applying $\langle P(X)|$ over particles $s+1, \ldots, s+m$ leads to

$$
\begin{aligned}
& \langle P(X) \mid \psi\rangle= \\
& =\sum_{i_{1}, \ldots, i_{s}, i_{s+m+1}, \ldots, i_{n}} \operatorname{tr}\left(\omega A_{i_{1}} \ldots A_{i_{s}} P(A) A_{i_{s+m+1}} \ldots A_{i_{n}}\right) \times \\
& \times\left|i_{1}, \ldots, i_{s}, i_{s+m+1}, \ldots, i_{n}\right\rangle .
\end{aligned}
$$

That is, we obtain a state similar to an $(n-m)$ site hMPS, but with an 'impurity' in the middle, namely the matrix polynomial $P(A)$. This notion of interacting with physical sites in order to engineer operators at the virtual level is actually the main idea behind measurement-based quantum computing on MPS [8].

Now, let $P(X)$ be a homogeneous MPI for dimension $D$ of degree $m$. Then, according to (5), $\langle P(X) \mid \psi\rangle=0$, i.e., the local operator $\langle P(X)|$ will have the property of annihilating any hMPS with bond dimension $D$ or smaller. Conversely, let $|P(X)\rangle$ be an $m$-particle vector with the property of annihilating all hMPS of bond dimension $D$ or smaller. Due to our freedom in choosing the boundary condition $\omega$, it is easy to see that $P(X)$ must necessarily be a MPI. We have just established that the local space spanned by hMPS is the orthogonal complement of the space of homogeneous MPIs for dimension $D$.

Note that, if $|\psi\rangle$ is a non-injective uniform TI MPS, then $\langle P(X) \mid \psi\rangle=0$ for all MPIs $P(X)$ of dimension $D-1$. This follows from the fact that the diagonal blocks of one of its matrix representations must have size $D-1$ or smaller.

The next question to answer is how big these two spaces are. Denote by $\mathcal{H}_{D, m}^{M P S}$ the $m$-local space spanned by hMPS of bond dimension $D$ of whatever size $n \geq m$, and call $\mathcal{H}_{D, m}^{M P I}$ the orthogonal complement of $\mathcal{H}_{D, m}^{M P S}$. Since MPIs of degree smaller than $2 D$ do not exist, we have that, for $m<2 D, \mathcal{H}_{D, m}^{M P I}=\{0\}$. Now, it is easy to see that $\mathcal{H}_{D, m}^{M P S}$ corresponds to $\operatorname{span}\{|\psi(\omega, A, m)\rangle$ : $\left.\omega, A_{1}, \ldots, A_{d} \in B\left(\mathbb{C}^{D}\right)\right\}$. Calling $\vec{a}$ the entries of the matrices $A$, we thus have that

$$
|\psi(\omega, A, m)\rangle=\sum_{w} w\left(\vec{a}, \omega_{i j}, m\right)\left|\phi_{w}\right\rangle,
$$

where the sum runs over all monomials $w$ of degree $m$ in $\vec{a}$ and degree 1 on the entries of $\omega$, and the vectors $\left\{\left|\phi_{w}\right\rangle\right\}_{w}$ do not depend on the particular values of $A, \omega$ (e.g.: the vector corresponding to the monomial $\omega_{11}\left(A_{1}\right)_{11}^{m}$ is $\left.|1\rangle^{\otimes m}\right)$. The above decomposition allows us to bound the dimensionality of $\mathcal{H}_{D, m}^{M P S}$ simply by counting the number of such monomials. The result is

$$
\operatorname{dim}\left(\mathcal{H}_{D, m}^{M P S}\right) \leq D^{2}\left(\begin{array}{c}
m+d D^{2}-1 \\
d D^{2}-1
\end{array}\right)
$$

where the $D^{2}$ factor stems from the number of entries of the boundary condition $\omega$. Relation (7) implies that the dimension of $\mathcal{H}_{D, m}^{M P S}$ increases polynomially with the system size $m$, contrarily to the total local space dimension, which increases as $d^{m}$. In the limit of high $m$, the space of MPIs is therefore exponentially bigger than $\mathcal{H}_{D, m}^{M P S}$.

Both the identification of $\mathcal{H}_{D, m}^{M P S}$ with the orthogonal complement of $\mathcal{H}_{D, m}^{M P I}$ and the polynomial bound on $\operatorname{dim}\left(\mathcal{H}_{D, m}^{M P S}\right)$ appear in Werner's encyclopaedic article [30].

In Appendix A we describe two efficient (i.e., with time complexity polynomial on $m$ ) algorithmic procedures to generate an orthonormal basis 


\begin{tabular}{|c|c|c|c|c|c|c|c|c|c|c|c|}
\hline$m$ & 5 & 6 & 7 & 8 & 9 & 10 & 11 & 12 & 13 & 14 & 15 \\
\hline$D=2$ & 30 & 53 & 88 & 139 & 210 & 306 & 432 & 594 & 798 & 1051 & 1360 \\
$D=3$ & 32 & 64 & 128 & 256 & 506 & 976 & 1820 & 3278 & 5700 & 9597 & $\times$ \\
$D=4$ & 32 & 64 & 128 & 256 & 512 & 1024 & 2048 & 3278 & 8192 & $\times$ & $\times$ \\
$D=\infty$ & 32 & 64 & 128 & 256 & 512 & 1024 & 2048 & 4096 & 8192 & 16384 & 32768 \\
\hline
\end{tabular}

Table 1: Dimension of the $m$-qubit subspace $\mathcal{H}_{D, m}^{M P S}$ for different values of the bond dimension $D$.

for $\mathcal{H}_{D, m}^{M P S}$. This allows us to ascertain the exact dimensionality of the spaces $\mathcal{H}_{D, m}^{M P S}$, for whatever values of $D, d, m$. Some results are presented in Table 1.

Let $h \in B\left(\mathcal{H}_{D, m}^{M P I}\right)$ be a self-adjoint operator acting on the space of MPIs of dimension $D$, and suppose that we integrate it in a $k$-local Hamiltonian $H$, with $k \geq m$. That is, suppose that the Hamiltonian of the system is $H^{\prime}=H+h$, with $H$ given by eq. (2).

The above discussion implies that hMPS of bond dimension $D$ or lower 'will not see' such a term, i.e., $H^{\prime}|\psi\rangle=H|\psi\rangle$ for all hMPS $|\psi\rangle=$ $|\psi(\omega, A, n)\rangle$, with $\omega, A \subset B\left(\mathbb{C}^{D}\right), n \geq m$. Elaborating on this, we find a limitation common to all hMPS-based variational methods for Hamiltonian minimization:

Proposition 1. Let $D>D^{\prime}>1$ be natural numbers. Then, for sufficiently large $n$, there exists a $O\left(D^{2}\right)$-local TI n-qubit Hamiltonian $H$ satisfying

$$
\begin{aligned}
& \langle H\rangle_{D^{\prime}-1}>\langle H\rangle_{D^{\prime}}=\langle H\rangle_{D^{\prime}+1}=\ldots \\
& \ldots=\langle H\rangle_{D-1}=\langle H\rangle_{D}>\langle H\rangle_{D+1}
\end{aligned}
$$

The result also holds when we restrict the Hamiltonian minimization to uniform TI MPS (with $\left.\omega=\mathbb{I}_{D}\right)$. Moreover, it can be extended to iMPS and TI Hamiltonians in the infinite spin chain. The Hamiltonian can also be taken $O(D)$-local at the cost of increasing the physical dimension of the particles.

See Appendix B for a proof.

A blind application of the proverbial method of minimizing a Hamiltonian via hMPS of increasing bond dimension until the sequence of energy values appears to converge hence risks getting stuck at a suboptimal point. Admittedly, the limitations implied by the Proposition do not pose a practical threat for usual studies of one-dimensional non-critical chains, since current hMPS-based algorithms allow reaching bond dimensions of order 100 in a normal computer (way beyond the locality of Hamiltonians of physical interest). This is no longer the case, though, for some other classes of TNS, for which we lack good optimization schemes and Proposition 1 also extends, see below. Note though that none of the arguments above apply to MPS or TNS with sitedependent tensors.

Let us conclude with a speculative thought: consider the evolution of hMPS under homogeneous Matrix Product Operators (MPO) rather than under local Hamiltonians. Since MPOs represent polynomials of arbitrarily high degree, in principle there could exist homogeneous MPOs with low bond dimension which nonetheless annihilate all hMPS of a moderately high bond dimension. That could jeopardize the performance of current algorithms to simulate time evolution in condensed matter systems, see [28].

Suppose now that we choose $P(X)$ in eq. (5) to be a central polynomial for dimension $D$. Then $P(A)=p(A) \mathbb{I}$, where $p(A)$ is a scalar. The state $|\psi\rangle$ will hence get projected into the state $p(A)\left|\psi^{\prime}\right\rangle$, with

$$
\begin{aligned}
\left|\psi^{\prime}\right\rangle=\sum_{\begin{array}{c}
i_{1}, \ldots, i_{s}, \\
i_{s+m+1}, \ldots, i_{n}
\end{array}} & \operatorname{tr}\left(\omega A_{i_{1}} \ldots A_{i_{s}} A_{i_{s+m+1}} \ldots A_{i_{n}}\right) \times \\
& \times\left|i_{1}, \ldots, i_{s}, i_{s+m+1}, \ldots, i_{n}\right\rangle .
\end{aligned}
$$

This is again a hMPS with the same boundary condition $\omega$ and matrices $A_{1}, \ldots, A_{d}$, but where $m$ particles are just missing.

Now, divide the space of $m$-degree homogeneous central polynomials into classes $[P]=P+$ $M P I$, i.e., two central polynomials $P_{1}, P_{2}$ belong to the same class if and only if $P_{1}-P_{2}$ is an MPI. These classes form a vector space $\mathcal{Q}_{D, m}$, the quotient space of $m$-degree homogeneous central polynomials by MPIs. Let $\left\{P_{i}(X)\right\}_{i}$ be a basis for $\mathcal{Q}_{D, m}$ and define the $m$-local operator $C \equiv \sum_{i}\left|\varphi_{i}\right\rangle\left\langle P_{i}(X)\right|$, where $\left\{\left|\varphi_{i}\right\rangle\right\}_{i}$ is any orthonormal set of $m$-particle states. The effect of $C$ over any hMPS of bond dimension $D$ or smaller is to project the $m$ particles where it acts into the pure state $|\varphi\rangle \equiv \sum_{i} p_{i}(A)\left|\varphi_{i}\right\rangle$, while the remaining particles end up in the state (9). It hence 'cuts' particles $s+1, \ldots, s+m$ off the chain and 'glues' the two ends back, see Figure 1.

This sort of operators will be called cut-andglue operators. Note that, after tracing out the 


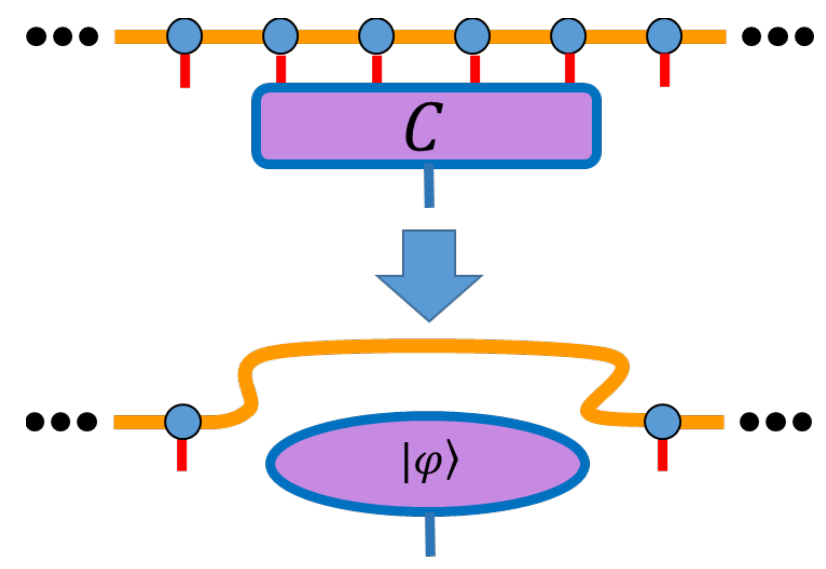

Figure 1: Action of a cut-and-glue operator $C$ over a MPS.

particles cut, a cut-and-glue operator acting over an iMPS leaves the spin chain in the same quantum state (modulo normalization).

To understand the significance of cut-and-glue operators, consider this: given an injective iMPS $|\psi\rangle$, with matrices $A_{1}, \ldots, A_{d}$, one can always find homogeneous polynomials $\left\{P_{i}(X)\right\}_{i}$ of degree $m$ such that $P_{i}(A) \propto \mathbb{I}_{D}$ for all $i$ (this follows from the injectivity condition). Hence the operator $\sum_{i}|i\rangle\left\langle P_{i}(X)\right|$ acting on $|\psi\rangle$ will produce a state of the form $\left(\sum_{i} p_{i}(A)|i\rangle\right)|\psi\rangle$. However, that same operator applied over an arbitrary iMPS $|\phi\rangle$ with matrices $B_{1}, \ldots, B_{d}$ will not produce a state proportional to $|\phi\rangle$, since $P_{i}(B) \not \subset \mathbb{I}_{D}$. Choosing $\left\{P_{i}(X)\right\}_{i}$ to be central polynomials, we make sure that $\sum_{i}|i\rangle\left\langle P_{i}(X) \mid \phi\right\rangle=\left(\sum_{i} p_{i}(A)|i\rangle\right)|\phi\rangle$ for all iMPS $|\phi\rangle$ of bond dimension $D$.

Finally, note that cut-and-glue operators for dimension $D$ annihilate all non-injective iMPS of bond dimension $D$, as well as all iMPS with lower bond dimension. This follows from the fact that any central polynomial $P$ for dimension $D$ evaluated on matrices $A=\left(A_{1}, \ldots, A_{d}\right)$ of size smaller than $D$ must equal zero. In effect, those can be embedded in $D \times D$ matrices as $\tilde{A}_{i} \equiv\left(\begin{array}{cc}A_{i} & 0 \\ 0 & 0\end{array}\right)$. Since by definition $P(\tilde{A})$ is proportional to the identity, the proportionality constant must be zero.

In Appendix $\mathrm{C}$ we sketch efficient procedures to generate a basis for $\mathcal{Q}_{D, m}$. Table 2 gives an idea of how the dimensionality of $\mathcal{Q}_{D, m}$ scales with the system size and the bond dimension in qubit ensembles. Surprisingly, it turns out that the dimensionality of $\mathcal{Q}_{2, m}$ in Table 2 follows the sequence of coefficients in the power series ex-

\begin{tabular}{|c|c|c|c|c|c|c|c|c|c|c|c|c|c|}
\hline$m$ & 3 & 4 & 5 & 6 & 7 & 8 & 9 & 10 & 11 & 12 & 13 & 14 & 15 \\
\hline$D=2$ & 0 & 1 & 2 & 6 & 10 & 20 & 30 & 50 & 70 & 105 & 140 & 196 & 252 \\
$D=3$ & 0 & 0 & 0 & 0 & 0 & 0 & 4 & 16 & 50 & 129 & 274 & 542 & $\times$ \\
\hline
\end{tabular}

Table 2: Dimensions of the $m$-qubit quotient spaces $\mathcal{Q}_{D, m}$ for different values of $m$ and bond dimensions $D=2,3$.

pansion of the Poincaré series $P\left(C_{2,2} ; t\right)$, which is sequence A096338 in the On-Line Encyclopedia of Integer Sequences [24]. We conjecture that the above observation holds true for entries beyond $m=15$ in Table 2 as well.

Both annihilation and cut-and-glue operators are important structural features of hMPS. Unfortunately, even for $D=2$ their implementation in the lab would require the ability to switch on non-trivial four-interaction terms. This is experimentally challenging, given that in many experimental setups only 2-local operators are accessible. Fortunately, there is a cleverer way to exploit our findings.

The notions of anihilation and cut-and-glue operators allow us to define a family of local operators $h$ whose average value is non-negative when computed with $n$-site MPS of bond dimension $D$ or smaller. Call $P$ the projector onto the space $\mathcal{H}_{D, n}^{M P S}$, and consider all $m$-local operators $h$ which satisfy:

$$
P h P=f+\sum_{j=1}^{n-1} P C_{j} g_{j} C_{j}^{\dagger} P,
$$

where $f \geq 0, C_{j}$ is a cut-and-glue operator acting non-trivially over particles $1, \ldots, j$ and $g_{j}$ is an entanglement witness [26] with respect to the partition $1, \ldots, j \mid j+1, \ldots, n$ (namely, $\left\langle g_{j}\right\rangle \geq 0$ for all quantum states separable with respect to the said partition). Clearly, $\langle h\rangle_{D} \geq 0$. Note that, if we drop the requirement of locality, one can construct operators of the form (10) for arbitrarily high bond dimensions just by combining known families of entanglement witnesses, central polynomials and matrix polynomial identities.

Given an arbitrary ( $k$-local) operator $H$, consider the problem of maximizing $\mu \in \mathcal{R}$ such that $H-\mu$ admits a decomposition of the form (10). Then, for any feasible $\mu,\langle H\rangle_{D} \geq \mu$. If the minimum eigenvalue of $H$ happens to be smaller than $\mu$, then $H-\mu$ can be regarded as a bond dimension witness: an expectation value for $H$ below $\mu$ would prove that the underlying quantum state of the system does not admit a hMPS model of 
bond dimension $D$.

Regretfully, the optimization proposed above requires an implicit characterization of entanglement witnesses, a problem known to be NP-hard [9]. A way out is to simply demand $g_{j}$ to belong to a class of entanglement witnesses which are easy to describe. An obvious choice is the set of all operators which are Positive (semidefinite) under Partial Transposition (PPT) [19]. With this restriction on $g_{j}$, the maximization of $\mu$ can be formulated as a semidefinite program (SDP), a class of convex optimization problems which can be solved efficiently [27].

The dual of this program would be an optimization over all quantum states $\rho \in B\left(\mathcal{H}_{D, m}^{M P S}\right)$ such that, for all $j, C_{j} \rho C_{j}^{\dagger}$ is PPT for the partition $1, \ldots, j \mid j+1, \ldots, n$. Since $\mathcal{H}_{D, m}^{M P S}$ grows polynomially with $m$, for small $D$ a normal computer can reach large values of $m$. Moreover, playing with the displacement operator, it is easy to derive a hierarchy of SDPs for the characterization of iMPS models.

Rather than describing these tools in detail [the reader can find a full description of the SDP programs in Appendix D], we will illustrate how these methods work with a practical example. Consider an $N$-site spin $1 / 2$ chain, and suppose that, via neutron interferometry, we estimate the expectation value of the XXX Heisenberg Hamiltonian

$$
H_{N}=\sum_{i=1}^{N-1} \frac{1}{4} \vec{\sigma}_{i} \cdot \vec{\sigma}_{i+1} .
$$

We wonder whether our experiment can be explained with a hMPS model of low bond dimension.

Take the number of particles in the chain to be small, say $N=7$. For low $N$, the minimum eigenvalue of $H_{N}$ can be computed exactly, and so we find that the minimum average energy per interaction term is $E \equiv \min \frac{1}{6}\left\langle H_{7}\right\rangle \approx-0.4727$. On the other hand, an SDP optimization over 7site normalized density matrices $\rho \in B\left(\mathcal{H}_{2,7}^{M P S}\right)$ satisfying $C_{j} \rho C_{j}^{\dagger}$, PPT for $j=5,6$ returns the greater value $E_{2} \equiv \frac{1}{6}\left\langle H_{7}\right\rangle_{2} \geq-0.4065$. This optimization, and all subsequent ones, was carried out with the SDP solver MOSEK [3]. The XXX Heisenberg Hamiltonian can thus be interpreted as a displaced bond dimension witness: an expectation value smaller than -0.4065 , within reach given the lower value of $E$, would signify that the state of the spin chain cannot have a hMPS model of bond dimension $D=2$.

Refuting $D=3$ hMPS models for $N=7$ is impossible with the tools developed so far, since $\mathcal{H}_{3, N}^{M P S}=\left(\mathbb{C}^{2}\right)^{\otimes N}$ and $\mathcal{Q}_{3, N}=\{0\}$, for $N=1, \ldots, 8$, see Tables 1 and 2 . To make matters worse, SDP optimizations for $N \geq 9$ are too memory-demanding for a normal computer. However, if we drop the PPT condition, the resulting SDP can be seen equivalent to projecting $\frac{1}{N-1} H_{N}$ on the subspace $\mathcal{H}_{3, N}^{M P S}$ and finding the minimum eigenvalue of the resulting operator. This simplified method allows us to reach greater values of $N$, at the price of losing robustness in our bounds. With this trick, for $N=13$ we obtain a bound $E_{3} \equiv \frac{1}{12}\left\langle H_{13}\right\rangle_{3} \geq-0.44958$, slightly bigger than the minimum energy density $E=-0.46044$ achievable.

For $N \gg 1$, we can take the system to be approximately translational invariant, so this time we want to refute iMPS models of low bond dimension for our system. For $D=d=2$ iMPS models, it can be shown that spin chains are symmetric under parity, and hence satisfy non-trivial linear constraints. However, if our experimental setup does not allow us to estimate quantities of the sort $\sum_{i=1}^{N-1}\left\langle\sigma_{i}^{s} \sigma_{i+1}^{t}\right\rangle$, with $t \neq s$, we must again rely on inequalities, in which case the XXX Heisenberg Hamiltonian can also serve as a witness. It is a standard result (see, e.g., [11]) that the ground state energy density $E=\lim _{N \rightarrow \infty} \frac{1}{N-1}\left\langle H_{N}\right\rangle$ of the infinite XXX Heisenberg model is given by $E=1 / 4-\ln (2) \approx$ -0.4431 .

Now, how to derive bounds for iMPS models? A possibility would be to compute the minimum expectation value of $\frac{1}{N-1}\left\langle H_{N}\right\rangle$ for high $N$ via the hMPS SDP relaxation used above. Intuitively, increasing values of $N$ should give better and better approximations to the optimal iMPS value for the energy density. We chose, though, to use the slightly better approximation of optimizing the value of $\frac{1}{4} \vec{\sigma}_{1} \cdot \vec{\sigma}_{2}$ over reduced density matrices subject to the constraints above and the extra condition $\rho \in \mathcal{S}_{D, N}$, where $\mathcal{S}_{D, N}$ denotes the span of the $N$-site reduced density matrices of iMPS of bond dimension $D$. This space can be characterized using similar techniques as the ones we applied to compute $\mathcal{H}_{D, m}^{M P S}$.

Optimizing over 8-site normalized reduced den- 
sity matrices $\rho \in B\left(\mathcal{H}_{2,8}^{M P S}\right)$ satisfying $C_{j} \rho C_{j}^{\dagger}$, PPT for $j=5,6,7$ and $\rho \in \mathcal{S}_{2,8}$ we find that $E_{2} \equiv \lim _{N \rightarrow \infty} \frac{1}{N-1}\left\langle H_{N}\right\rangle_{2} \geq-0.3378$. An average energy lower than the last value will hence refute all iMPS models of bond dimension $D=2$.

This example is very illuminating in that it allows to appreciate the relevance of cut-and-glue operators for this class of optimizations. For, if we drop the PPT conditions above, the lower bound on $E_{2}$ output by the computer decreases to -0.4246 . This is still bigger than $E$, and so it also defines a bond dimension witness. However, it is one order of magnitude less robust than the previous one, and so its violation is more challenging from an experimental point of view.

As a final example, we consider the MajumdarGhosh Hamiltonian [14]:

$$
H_{M G}=\sum_{i=1}^{N-2} \frac{1}{8}\left(2 \vec{\sigma}_{i} \vec{\sigma}_{i+1}+\vec{\sigma}_{i} \vec{\sigma}_{i+2}\right) .
$$

The expectation value of this operator can also be estimated experimentally via neutron diffusion. In the thermodynamical limit $N \rightarrow \infty$, the minimum energy density of $H_{M G}$ is $E=-\frac{3}{8}=$ -0.375 , achievable with an iMPS of bond dimension $D=3[20]$. In contrast, an $8^{\text {th }}$-order SDP relaxation over iMPS of bond dimension $D=2$ gives $E_{2} \geq-0.2593$. We have just derived a bond dimension witness with a large gap between iMPS models with $D=2$ and $D=3$. On the negative side, though, our lower bound for $E_{2}$ is significantly lower than the best upper bound $E_{2} \leq-0.125$ we found using the Amoeba variational method [31].

Note that the former SDP methods can be easily turned into feasibility tests. Indeed, determining the existence of a state with the properties above compatible with some partial information we may hold about the quantum state of the spin chain (such as, e.g., the average value of a number of 2-local observables) can also be cast as an SDP. This procedure can help an experimentalist to refute the existence of a hMPS or iMPS model for the state he/she prepared in the lab, without the need of guessing the 'right' bond dimension witness to do the job.

An immediate question is whether the SDP hierarchy of relaxations for iMPS models sketched above is complete, in the sense that it allows us to detect any state lacking an iMPS model by taking $N$ sufficiently large (the SDP relaxation for general hMPS is clearly not complete). In this regard, notice that $\mathcal{H}_{1, N}^{M P S}$ corresponds to the symmetric space of $N$ particles, and $\mathcal{Q}_{1, N}=$ $\mathcal{H}_{1, N}^{M P S}$. Hence for $D=1$ the hierarchy reduces to just imposing that the overall state is symmetric and PPT with respect to any bipartition. This is actually the Doherty-Parrilo-Spedalieri (DPS) method for entanglement detection [4], and convergence follows from the quantum de Finetti theorem [12]. Similarly, $\mathcal{H}_{\infty, N}^{M P S}=\mathbb{C}^{d^{N}}, \mathcal{Q}_{\infty, N}=\{0\}$ and $\mathcal{S}_{\infty, N}$ is the span of all TI states. For $D=\infty$ the hierarchy is therefore computing the minimum expectation value of a Hamiltonian term over $N$-site states whose $N-1$-site reduced density matrices coincide whenever we remove the first or the last site. This is essentially a reformulation of Anderson's approximation [2], where convergence is also known to hold. One would be tempted to claim that our SDP hierarchy should converge as well for all intermediate values of $D$, but we leave this matter open.

\section{Applications for optimizations over finite dimensional quantum correlations}

In $[15,16]$, a hierarchy of SDP relaxations is presented to characterize the statistics of finitedimensional quantum systems. This hierarchy relies on the notion of moment matrices. Given a quantum system in state $\sigma \in B\left(\mathbb{C}^{D}\right)$, with (self-adjoint) operators $X_{1}, \ldots, X_{d-1} \subset B\left(\mathbb{C}^{D}\right)$, its $n^{\text {th }}$ order moment matrix $M$ is a matrix whose rows and columns are labeled by monomials $u$ of $X_{1}, \ldots, X_{d-1}$ of degree smaller than or equal to $n$, with entries given by $M_{u, v}=\operatorname{tr}\left(u^{\dagger}(X) \sigma v(X)\right)$. In $[15,16]$, it is proposed to relax the requirement of $M$ admitting a quantum representation by demanding $M \geq 0$ and $M \in \mathcal{M}_{D}$, where $\mathcal{M}_{D}$ denotes the space spanned by moment matrices with quantum representations of dimension $D$.

A disadvantage of this method is that, for fixed $d, D$, the complexity of implementing the hierarchy increases exponentially with the index $n$ of the relaxation. In the following, we show that every $n^{\text {th }}$-order moment matrix with a quantum representation of dimension $D$ can be interpreted as a conic combination of $n$-site hMPS with bond dimension $D$. This will allow us to devise a hMPS-based algorithm that carries out an improved version of the $n^{t h}$-order relaxation 
described in $[15,16]$ in time polynomial in $n$.

Define $X_{d} \equiv \mathbb{I}_{D}$, and consider vectors of $d$ indices with values in $\{1, \ldots, d\}$. Then, for any index vector $\vec{i} \in\{1, \ldots, d\}^{k}$, we can associate the monomial $u(X)_{\vec{i}} \equiv X_{i_{1}} \ldots X_{i_{n}}$. This procedure gives an over-representation of the set of monomials of degree smaller than or equal to $n$.

Now suppose that, by repeating rows and columns, we enlarge the $n^{\text {th }}$-order moment matrix $M$ of the system to an $d^{k} \times d^{k}$ matrix $\bar{M}$ such that $\langle\vec{i}|\bar{M}| \vec{j}\rangle=M_{u_{\vec{i}}, v_{\vec{j}}}$, with $|\vec{i}\rangle=\left|i_{1}\right\rangle \ldots\left|i_{n}\right\rangle$ and similarly for $|\vec{j}\rangle$. The 'enhanced' moment matrix $\bar{M}$ can then be written as

$$
\bar{M}_{k}=\sum_{\vec{i}, \vec{j}} \operatorname{tr}\left(X_{i_{n}}^{\dagger} \ldots X_{i_{1}}^{\dagger} \sigma X_{j_{1}} \ldots X_{j_{n}}\right)|\vec{i}\rangle\langle\vec{j}| .
$$

This is just the transpose of a conic combination of hMPS of bond dimension $D$; much like eq. (3), but without the condition $\sum_{i} A_{i}^{\dagger} \sigma A_{i}=\sigma$. As such, its support is contained in $\mathcal{H}_{D, n}^{M P S}$; more precisely, in the analog set for hMPS with $X_{d}=\mathbb{I}$ plus any other extra restriction in the variables $X$. Hence $\bar{M}$ can be fully specified by a number of parameters polynomial in $n$.

Most interestingly, one can compute special cut-and-glue operators $C$ for this kind of hMPS. The convergence of the scheme can therefore be boosted by demanding extra positive semidefinite constraints such as $\left(C_{j} \bar{M} C_{j}^{\dagger}\right)^{T_{j}} \geq$ 0. 'Localizing matrices' of the form $M_{u, v}^{q}=$ $\sum_{\vec{i}, \vec{j}} \operatorname{tr}\left(X_{\vec{i}}^{\dagger} \sigma X_{\vec{j}} q(X)\right)|\vec{i}\rangle\langle\vec{j}|$, defined in [16] to model semi-algebraic conditions of the sort $q(X) \geq 0$, can be treated in a similar way.

\section{Extension to Projected Entangled Pairs States}

MPS can be understood as elements of a larger class of TNS called Projected Entangled Pairs States (PEPS) [23]. Such states are used to approximate the low energy sector of local Hamiltonians describing particles sited in square lattices of arbitrary spatial dimensions. While MPS are defined via tensors with one physical index $(i=1, \ldots, d)$ and two bond indices (the column and row indices of the matrices $\left.A_{1}, \ldots, A_{d}\right), N$ dimensional PEPS are defined via contractions of tensors with 1 physical index and $2 N$ bond indices. MPS can therefore be regarded as onedimensional PEPS. For illustration, in Figure 2

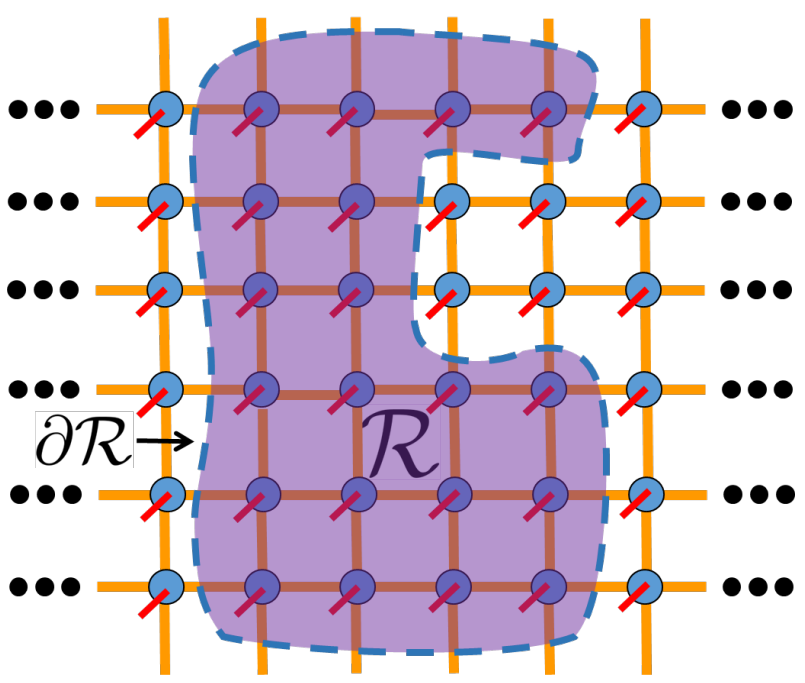

Figure 2: The region $\mathcal{R}$ in the two-dimensional lattice is marked in purple. Its boundary $\partial \mathcal{R}$ (dashed line) corresponds to all broken links of the repeated tensor.

the tensors of a two-dimensional PEPS are represented by circles, while physical [bond] indices are denoted by red [orange] lines. If we assume that all such tensors are equal, we arrive at the notion of homogeneous PEPS (hPEPS). It is natural to ask whether some of the structural features we derived for hMPS also extend to hPEPS of higher spatial dimension.

Consider the space spanned by hPEPS of bond dimension $D$ and physical dimension $d$ in a given region $\mathcal{R}$ of the lattice, with boundary $\partial \mathcal{R}$, see Figure 2. As with hMPS, we can express any hPEPS in $\mathcal{R}$ as in (6), where each monomial $w$ has degree 1 on the boundary condition and degree $|\mathcal{R}|$ on the tensor $A$ generating the hPEPS. The number of such monomials is $D^{|\partial \mathcal{R}|}\left(\begin{array}{c}|\mathcal{R}|+d D^{2}-1 \\ d D^{2}-1\end{array}\right)$, and hence, for fixed $d, D$, the local space spanned by hPEPS is upper bounded by $D^{|\partial \mathcal{R}|} \operatorname{poly}(|\mathcal{R}|)$. This bound must be compared with the total dimensionality of the physical space in $\mathcal{R}$, namely, $d^{|\mathcal{R}|}$. Provided that $d^{|\mathcal{R}|}>\operatorname{poly}(|\mathcal{R}|) D^{|\partial \mathcal{R}|}$, we will find non-trivial operators in $\mathcal{R}$ which will annihilate all hPEPS with bond dimension $D$ or smaller. If the spatial dimension of the lattice is $N$, taking $\mathcal{R}$ to be a hypercube of size $L$, with volume $|\mathcal{R}|=L^{N}$ and surface area $|\partial \mathcal{R}|=2 N L^{N-1}$, this will happen for $L$ high enough.

We have just proven the existence of tensor polynomial identities, i.e., local vectors $|\phi\rangle$ which annihilate all PEPS of bond dimension $D$. Given that generic hPEPS are the ground state of a 
unique parent Hamiltonian, it is easy to prove a weaker version of Proposition 1 for hPEPS of arbitrary spatial dimension. Namely, that the chain of identities will break at some point beyond $\langle H\rangle_{D}$ (not necessarily at $D+1$ ). Let us remark that, contrarily to hMPS variational algorithms, current tools for optimizations over hPEPS do not allow the user to reach high bond dimensions. Hence, even if the Hamiltonian is $k$-local, it may be that we can just compute the values $\left\{\langle H\rangle_{D}: D \ll k\right\}$. In such a predicament we may be eager to believe that the last estimation is a good approximation to the ground state energy, if the corresponding optimizations over lower bond dimensions returned similar results... and we could be wrong, as the arguments above show.

\section{Conclusion}

In this work, we have presented two highly nontrivial structural properties of hMPS, namely, the existence of annihilation and cut-and-glue operators. We used these notions to prove several results concerning the limitations of the hMPS approximation. Along the way, we raised a number of important open questions.

First, it would be desirable to find closed formulas for the dimensionalities of $\mathcal{H}_{D, m}^{M P S}$ and $\mathcal{Q}_{D, m}$. Even though we have efficient methods to calculate these exactly, large values of $m$ require a considerable amount of computational time (hence the missing entries in Tables 1, 2). Perhaps the connection with the Poincare series $P\left(C_{2,2} ; t\right)$ can be exploited in this regard.

Another interesting problem is whether our SDP hierarchy of relaxations to refute iMPS models is complete or can be further improved.

Regarding completeness, for $D=1$ the proof of convergence follows from the quantum de Finetti theorem [12]. For $D=\infty$, the hierarchy is just a reformulation of Anderson's approximation [2], whose convergence was established long ago. A convergence proof for all other values of $D$ would not only provide us with an alternative definition of iMPS, but most likely would involve an intermediate result of depth comparable to the quantum de Finetti theorem.

As for improvement, a promising avenue to boost the speed of convergence of the hierarchy is to incorporate to our codes entropic constraints of the form $S\left(\rho_{1, \ldots, k}\right) \geq S\left(\rho_{1, \ldots, k-1}\right)$ for $2 \leq k \leq n$, as in [22]. These hold for any TI state; and, in particular, for iMPS. Although not reducible to SDPs, the corresponding problems can nonetheless be attacked with the tools of convex optimization theory. Considering the space spanned by several copies of a hMPS should also help.

Admittedly, it is difficult to believe that these ideas can ever lead to non-trivial restrictions for hMPS models with $D>4$. Devising new tools for the characterization of hMPS models of high bond dimension is hence an important matter.

Finally, it is intriguing whether the analogs of cut-and-glue operators for hMPS also exist for hPEPS of higher dimension. The action of such local operators over an arbitrary hPEPS would be to project the region where they act into a pure state and interconnect the links of the particles in the boundary. Appropriately tamed, such operators would allow transforming tensor network states of different type into each other by means of fixed (i.e., state-independent) local operations, very much like graph states transform into each other [10]. In this case, however, computational explorations face the exponential complexity of characterizing the space spanned by hPEPS of dimensions two and higher.

Acknowledgements We thank Andreas Winter for proving that the dimensionality of $\mathcal{H}_{D, m}^{M P S}$ grows polynomially with $m$. T.V. acknowledges the support of the National Research, Development and Innovation Office NKFIH (Grant Nos. K111734 and KH125096). This work was not funded by the European Research Council.

\section{References}

[1] Dorit Aharonov, Daniel Gottesman, Sandy Irani, and Julia Kempe. The power of quantum systems on a line. Communications in Mathematical Physics, 287(1):4165, jan 2009. DOI: 10.1007/s00220-0080710-3. URL https://doi.org/10.1007\% 2Fs00220-008-0710-3.

[2] P. W. Anderson. Limits on the energy of the antiferromagnetic ground state. Physical Review, 83(6):1260-1260, sep 1951. DOI: 10.1103/physrev.83.1260. URL https:// doi.org/10.1103\%2Fphysrev. 83.1260.

[3] MOSEK ApS. The MOSEK optimization 
toolbox for MATLAB manual. Version 7.1 (Revision 28)., 2015. URL http://docs. mosek.com/7.1/toolbox/index.html.

[4] A. C. Doherty, Pablo A. Parrilo, and Federico M. Spedalieri. Distinguishing separable and entangled states. Physical Review Letters, 88(18), apr 2002. DOI: 10.1103/physrevlett.88.187904. URL https://doi.org/ 10.1103\%2Fphysrevlett.88.187904.

[5] Glen Evenbly and Guifre Vidal. Quantum criticality with the multi-scale entanglement renormalization ansatz. In Springer Series in Solid-State Sciences, pages 99-130. Springer Berlin Heidelberg, 2013. DOI: 10.1007/9783-642-35106-8_4. URL https://doi.org/ 10.1007\%2F978-3-642-35106-8_4.

[6] M. Fannes, B. Nachtergaele, and R. F. Werner. Finitely correlated states on quantum spin chains. Communications in Mathematical Physics, 144(3):443-490, mar 1992. DOI: 10.1007/bf02099178. URL https:// doi.org/10.1007\%2Fbf02099178.

[7] Edward Formanek. The Polynomial Identities and Variants of $n \times n$ Matrices. American Mathematical Society, jan 1991. DOI: 10.1090/cbms/078. URL https://doi. org $/ 10.1090 \% 2 \mathrm{Fcbms} \% 2 \mathrm{~F} 078$.

[8] D. Gross, J. Eisert, N. Schuch, and D. Perez-Garcia. Measurement-based quantum computation beyond the one-way model. Physical Review A, 76(5), nov 2007. DOI: $10.1103 /$ physreva.76.052315. URL https://doi.org/10.1103\%2Fphysreva. 76.052315 .

[9] Leonid Gurvits. Classical complexity and quantum entanglement. Journal of Computer and System Sciences, 69(3):448-484, nov 2004. DOI: 10.1016/j.jcss.2004.06.003. URL https://doi.org/10.1016\%2Fj. jcss.2004.06.003.

[10] M. Hein, J. Eisert, and H. J. Briegel. Multiparty entanglement in graph states. Physical Review A, 69(6), jun 2004. DOI: 10.1103/physreva.69.062311. URL https://doi.org/10.1103\%2Fphysreva. 69.062311.

[11] Michael Karbach, Kun $\mathrm{Hu}$, and Gerhard Muüller. Introduction to the bethe ansatz II. Computers in Physics, 12(6):565, 1998. DOI: 10.1063/1.168740. URL https://doi. org $/ 10.1063 \% 2 \mathrm{~F} 1.168740$.
[12] Robert König and Renato Renner. A de finetti representation for finite symmetric quantum states. Journal of Mathematical Physics, 46(12):122108, dec 2005. DOI: 10.1063/1.2146188. URL https://doi. org/10.1063\%2F1.2146188.

[13] Michael Levin and Cody P. Nave. Tensor renormalization group approach to two-dimensional classical lattice models. $\quad$ Physical Review Letters, 99 (12), sep 2007. DOI: 10.1103/physrevlett.99.120601. URL https://doi.org/ 10.1103\%2Fphysrevlett.99.120601.

[14] Chanchal K. Majumdar and Dipan K. Ghosh. On next-nearest-neighbor interaction in linear chain. i. Journal of Mathematical Physics, 10(8):1388-1398, aug 1969. DOI: 10.1063/1.1664978. URL https:// doi.org/10.1063\%2F1. 1664978.

[15] Miguel Navascués and Tamás Vértesi. Bounding the set of finite dimensional quantum correlations. Physical Review Letters, 115(2), jul 2015. DOI: $\quad 10.1103$ / physrevlett.115.020501. URL https://doi.org/10.1103\% 2Fphysrevlett.115.020501.

[16] Miguel Navascués, Adrien Feix, Mateus Araújo, and Tamás Vértesi. Characterizing finite-dimensional quantum behavior. Physical Review A, 92(4), oct 2015. DOI: $10.1103 /$ physreva.92.042117. URL https://doi.org/10.1103\%2Fphysreva. 92.042117 .

[17] Roberto Oliveira and Barbara M. Terhal. The complexity of quantum spin systems on a two-dimensional square lattice. Quant. Inf, Comp., 8, 2008.

[18] Román Orús. A practical introduction to tensor networks: Matrix product states and projected entangled pair states. Annals of Physics, 349:117-158, oct 2014. DOI: 10.1016/j.aop.2014.06.013. URL https:// doi.org/10.1016\%2Fj . aop. 2014.06.013.

[19] Asher Peres. Separability criterion for density matrices. Physical Review Letters, 77(8): 1413-1415, aug 1996. DOI: 10.1103/physrevlett.77.1413. URL https://doi.org/ 10.1103\%2Fphysrevlett. 77.1413.

[20] D. Perez-García, F. Verstraete, M. M. Wolf, and J.I. Cirac. Matrix product state rep- 
resentations. Quantum Inf. Comput., 7:401, sep 2007.

[21] Ho N. Phien, Johann A. Bengua, Hoang D. Tuan, Philippe Corboz, and Román Orús. Infinite projected entangled pair states algorithm improved: Fast full update and gauge fixing. Physical Review B, 92(3), jul 2015. DOI: 10.1103/physrevb.92.035142. URL https://doi.org/ 10.1103\%2Fphysrevb. 92.035142 .

[22] David Poulin and Matthew B. Hastings. Markov entropy decomposition: A variational dual for quantum belief propagation. Physical Review Letters, 106(8), feb 2011. DOI: $\quad$ 10.1103/physrevlett.106.080403. URL https://doi.org/10.1103\% 2Fphysrevlett.106.080403.

[23] Norbert Schuch, Ignacio Cirac, and David Pérez-García. PEPS as ground states: Degeneracy and topology. Annals of Physics, 325(10):2153-2192, oct 2010. DOI: 10.1016/j.aop.2010.05.008. URL https:// doi.org/10.1016\%2Fj . aop. 2010.05.008.

[24] Neil J. A. Sloane. The on-line encyclopedia of integer sequences. In Towards Mechanized Mathematical Assistants, pages 130-130. Springer Berlin Heidelberg. DOI: 10.1007/978-3-540-730866_12. URL https://doi.org/10.1007\% 2F978-3-540-73086-6_12.

[25] Stellan Östlund and Stefan Rommer. Thermodynamic limit of density matrix renormalization. Physical Review Letters, 75(19): 3537-3540, nov 1995. DOI: 10.1103/physrevlett.75.3537. URL https://doi.org/ 10.1103\%2Fphysrevlett.75. 3537.

[26] Barbara M. Terhal. Bell inequalities and the separability criterion. Physics Letters A, 271 (5-6):319-326, jul 2000. DOI: 10.1016/s03759601(00)00401-1. URL https://doi.org/ 10. $1016 \% 2 \mathrm{Fs} 0375-9601 \% 2800 \% 2900401-1$.

[27] Lieven Vandenberghe and Stephen Boyd. Semidefinite programming. SIAM Review, 38(1):49-95, mar 1996. DOI: 10.1137/1038003. URL https://doi.org/ $10.1137 \% 2 \mathrm{~F} 1038003$.

[28] F. Verstraete, J. J. García-Ripoll, and J. I. Cirac. Matrix product density operators: Simulation of finite-temperature and dissipative systems. Physical Review Letters, 93(20), nov 2004. DOI: 10.1103/phys- revlett.93.207204. URL https://doi.org/ 10.1103\%2Fphysrevlett.93.207204.

[29] F. Verstraete, V. Murg, and J.I. Cirac. Matrix product states, projected entangled pair states, and variational renormalization group methods for quantum spin systems. Advances in Physics, 57(2):143-224, mar 2008. DOI: 10.1080/14789940801912366. URL https://doi.org/10.1080\% 2F14789940801912366.

[30] R.F. Werner. Finitely correlated states. In Encyclopedia of Mathematical Physics, pages 334-340. Elsevier, 2006. DOI: $10.1016 /$ b0-12-512666-2/003795. URL https://doi.org/10.1016\% 2Fb0-12-512666-2\%2F00379-5.

[31] Eric Ziegel, William Press, Brian Flannery, Saul Teukolsky, and William Vetterling. Numerical recipes: The art of scientific computing. Technometrics, 29(4):501, nov 1987. DOI: $10.2307 / 1269484$. URL https://doi. org $/ 10.2307 \% 2 \mathrm{~F} 1269484$.

\section{A Exploring $\mathcal{H}_{D, m}^{M P S}$}

Except for $D=1$, the upper bound defined by eq. (7) in the main text is not tight, the reason being that the vectors $\left\{\left|\phi_{w}\right\rangle\right\}_{w}$ are linearly dependent. This is (partly) due to the fact that different values of $A, \omega$ may correspond to the same hMPS. Indeed, take $D=d=2$, and note that, for any matrix $S \in B\left(\mathbb{C}^{D}\right)$, the transformation $\omega \rightarrow S^{-1} \omega S, A_{i} \rightarrow S^{-1} A_{i} S$ leaves the state in eq. (1) of the main text invariant. In particular, we can choose $S$ to diagonalize $A_{1}$. Applying furthermore a diagonal transformation $T=\operatorname{diag}(a, b)$ we can enforce that the off-diagonal elements of $A_{2}$ are equal. With this parametrization, $A$ is specified by just 5 parameters, and so the dimensionality of $\mathcal{H}_{2, m}^{M P S}$ is expected to grow as $O\left(m^{4}\right)$, rather than $O\left(m^{7}\right)$, as the formula (7) in the text suggests.

Determining the exact dimensionality of $\mathcal{H}_{D, m}^{M P S}$ and deriving an orthonormal basis for this subspace can be done via two different procedures. First, given an arbitrary weight $w(\omega, A)>0$, we will call polynomial MPS any $m$-site states of the form 


$$
\left|f^{M P S}\right\rangle \equiv \int d \omega d A w(\omega, A) f(\omega, A)^{*}|\psi(\omega, A, m)\rangle,
$$

where $f(\omega, A)$ is a homogeneous polynomial of the components of $\omega$ (with degree 1) and $A$ (with degree $m$ ). A comfortable possibility is to take $\omega, A$ real and the trivial weight $w(\omega, A)=1$. Defining $t \equiv \operatorname{dim}\left(\mathcal{H}_{D, m}^{M P S}\right)$, our task is to find a set of polynomials $\left\{f_{i}\right\}_{i=1}^{t}$ such that $\left\langle f_{i}^{M P S} \mid f_{j}^{M P S}\right\rangle=\delta_{i j}$ and $\operatorname{span}\left\{\left|f_{i}^{M P S}\right\rangle\right\}=$ $\mathcal{H}_{D, m}^{M P S}$. This can be seen equivalent to diagonalizing the kernel

$$
\begin{aligned}
& K\left(A, \omega, A^{\prime}, \omega^{\prime}\right) \equiv \\
& \left\langle\psi\left(\omega^{\prime}, A^{\prime}\right) \mid \psi(\omega, A)\right\rangle w(\omega, A) w\left(\omega^{\prime}, A^{\prime}\right)= \\
& =\operatorname{tr}\left\{\left(\sum_{i=1}^{d} \bar{A}_{i}^{\prime} \otimes A_{i}\right)^{N}\left(\bar{\sigma}^{\prime} \otimes \sigma\right)\right\} w(\omega, A) w\left(\omega^{\prime}, A^{\prime}\right),
\end{aligned}
$$

on a basis of homogeneous polynomials of degree 1 in $\omega$ and $m$ in the entries of $A$ and taking the eigenvectors (polynomials) with non-zero eigenvalue.

Alternatively, we can simply sequentially generate real random $D \times D$ matrices $\omega^{j}, A_{1}^{j}, \ldots, A_{d}^{j}$ and use them to define the sequence of random hMPS $\left(\left|\psi\left(\omega^{j}, A^{j}, m\right)\right\rangle\right)_{j}$. Exploiting the fact that the overlap between two hMPS can be computed efficiently, one can apply a Gram-Schmidt process to the previous sequence of hMPS, thus obtaining an orthonormal basis for $\mathcal{H}_{D, m}^{M P S}$, whose elements are finite linear combinations of hMPS.

\section{B Proof of Proposition 1}

The goal of this section is to prove Proposition 1 in the main text, which reads:

Proposition 2. Let $D>D^{\prime}>1$ be natural numbers. Then, for any $N$, there exists an $O\left(D^{2}\right)$ local TI n-qubit Hamiltonian $H$, with $n>N$, satisfying

$$
\begin{aligned}
& \langle H\rangle_{D^{\prime}-1}>\langle H\rangle_{D^{\prime}}=\langle H\rangle_{D^{\prime}+1}=\ldots \\
& \left.\ldots=\langle H\rangle_{D-1}=\langle H\rangle_{D}\right\rangle\langle H\rangle_{D+1} .
\end{aligned}
$$

For the proof we need two intermediate results, namely:
1. There exist TI $O(D)$-local Hamiltonians of arbitrarily many particles whose unique ground state is a hMPS of bond dimension $D$. This is proven in Section B.1.

2. For any $D>1$, there exists a bivariate noncommutative homogeneous polynomial $F\left(X_{1}, X_{2}\right)$ of degree $O\left(D^{2}\right)$ that is a MPI for matrices of size $D-1 \times D-1$, but not for matrices of size $D \times D$. This will be proven in Section B.2.

These two results will be combined to demonstrate the Proposition in Section B.3.

\section{B.1 Parent Hamiltonians and MPIs}

A set of matrices $A_{1}, \ldots, A_{d} \in B\left(\mathbb{C}^{D}\right)$ satisfies the injectivity condition if there exists $k$ such that the products $\left\{A_{i_{1}} \ldots A_{i_{k}}\right\}$ span all of $B\left(\mathbb{C}^{D}\right)$. From [20], we know that any $n$-site uniform TI MPS $|\psi\rangle$ whose matrices satisfy the injectivity condition for some order $k$ can be seen as the unique ground state of a (TI) $2 k$-local Hamiltonian (provided that $n \geq 2 k$ ). The latter is called the parent Hamiltonian of $|\psi\rangle$.

We will next prove that, for any $D$, there exist matrices $B_{1}, B_{2} \in B\left(\mathbb{C}^{D}\right)$ which satisfy the injectivity condition for $k=O(D)$. With the above, this will imply that, for any bond and physical dimensions $D, d$ and any system size $n$, there exists an $n$-site TI MPS which arises as the unique ground state of a $O(D)$-local TI Hamiltonian.

Let $d=2$, and consider the matrices

$$
B_{1}=\sum_{j=1}^{D} j|j\rangle\left\langle j\left|, B_{2}=\frac{1}{D} \sum_{i, j=1}^{D}\right| i\right\rangle\langle j|
$$

Note that we can express the projectors $\{|i\rangle\langle i|\}_{i=1}^{D}$ as linear combinations of $\left\{B_{1}^{p}: p=1, \ldots, D\right\}$. Since $B_{2}^{s}=B_{2}$ for any $s \geq 1$, this implies that linear combinations of the (degree $2 D+1$ ) products $B_{1}^{p} B_{2}^{2 D-p-q+1} B_{1}^{q}$ can generate the matrices $|i\rangle\left\langle i\left|B_{2}\right| j\right\rangle\left\langle j\left|=\frac{1}{D}\right| i\right\rangle\langle j|$, which span $B\left(\mathbb{C}^{D}\right)$.

\section{B.2 MPls for dimension $D-1$ which cease to be identities in dimension $D$}

In this section we will prove that, for any $D$, there exists a polynomial $F\left(X_{1}, X_{2}\right)$ of degree $O\left(D^{2}\right)$ which is a MPI for dimension $D-1$, but not for dimension $D$. 
Choose $B_{1}, B_{2} \in B\left(\mathbb{C}^{D}\right)$ as in (17). As proven in Appendix B.1, there exist homogeneous polynomials $\left\{P_{j}(X)\right\}_{j}$ of degree $O(D)$ such that $P_{1}(B)=|1\rangle\left\langle 1\left|, P_{2}(B)=\right| 1\right\rangle\langle 2|, \ldots, P_{2 D-3}(B)=$ $|D-1\rangle\left\langle D-1\left|, P_{2 D-2}(B)=\right| D-1\right\rangle\langle D|$. Note that the matrices $\left\{P_{j}(B)\right\}_{j}$ have the peculiarity that the only permutation of them which does not vanish is $P_{1}(B) P_{2}(B) \ldots P_{2(D-1)}(B)$. Hence, by construction, the standard polynomial [eq. 4 in the main text] with $N=2(D-1)$, applied to the tuple $Y=\left(P_{1}(B), P_{2}(B), \ldots\right)$, results in a non-zero value. That is, the $O\left(D^{2}\right)$ degree homogeneous polynomial $P\left(X_{1}, X_{2}\right) \equiv$ $F_{2(D-1)}\left(P_{1}(X), \ldots, P_{2(D-1)}(X)\right)$, while being an MPI for $D-1 \times D-1$ matrices, is not an MPI for dimension $D$.

\section{B.3 Putting all together}

Now we are ready to prove Proposition 1.

Proof. Take a TI $n$-qubit MPS $|\psi\rangle$ with bond dimension $D^{\prime}$, and build its TI parent Hamiltonian $H$ [20]. Such is a $k$-local operator with the properties $H \geq 0, H|\psi\rangle=0$ and $|\psi\rangle$ being the only ground state of $H$. From Section B.1 we know that $|\psi\rangle$ can be chosen injective and such that $H$ has interaction strength $k=O\left(D^{\prime}\right)$. Since $|\psi\rangle$ is injective, it cannot be expressed as a hMPS of bond dimension $D^{\prime}-1$ (because, e.g., $|\psi\rangle$ has Schmidt rank greater than $\left.D^{\prime}-1\right)$. It follows that $\left.\langle H\rangle_{D^{\prime}-1}\right\rangle\langle H\rangle_{D^{\prime}}=0$.

Now, for $m$ high enough, choose $h \in B\left(\mathcal{H}_{D, m}^{M P I}\right)$, $h>0$, with $h \notin B\left(\mathcal{H}_{D+1, m}^{M P I}\right)$. From Section B.2 we know that, no matter the value of the physical dimension, there exists such an operator with $m=O\left(D^{2}\right)$. Given $h$, define the family of $O\left(D^{2}\right)$-local TI Hamiltonians $H_{\lambda}=$ $H-\lambda \sum_{i=1}^{n} \tau^{i}(h)$, where $\tau$ is the translation operator. By construction, $H_{\lambda}|\psi\rangle=H|\psi\rangle$ for all hMPS of bond dimension smaller than or equal to $D$, and so the equalities in eq. (8) are satisfied. On the other hand, for $\lambda$ high enough, $\left\langle H_{\lambda}\right\rangle_{D+1}<0$.

Note that, if we are entitled to play with the physical dimension of the system, we do not need to invoke Section B.2 at all. Indeed, it suffices to set $d=2 D$ and take $h$ to be the standard identity for dimension $D$ (which, having degree $2 D$, cannot be an MPI for dimension $D+1$ ). The corresponding family of Hamiltonians $H_{\lambda}$ would then be $O(D)$-local, rather than $O\left(D^{2}\right)$-local.

\section{Characterizing $\mathcal{Q}_{D, m}$}

Viewed as a subspace of $\left(\mathbb{C}^{d}\right)^{\otimes m}$, the space of central polynomials $\mathcal{H}_{D, m}^{C P}$ corresponds to the orthogonal complement of

$$
\begin{aligned}
& \mathcal{H}_{D, m}^{M P S,[],}=\operatorname{span}\left\{\sum_{i_{1}, \ldots, i_{m}} \operatorname{tr}\left(\omega\left[B, A_{i_{1}}, \ldots, A_{i_{m}}\right]\right)\left|i_{1}, \ldots, i_{m}\right\rangle\right\}= \\
& =\operatorname{span}\left\{\sum_{i_{1}, \ldots, i_{n}} \operatorname{tr}\left([\omega, B] A_{i_{1}}, \ldots, A_{i_{m}}\right)\left|i_{1}, \ldots, i_{m}\right\rangle\right\}= \\
& =\operatorname{span}\{|\psi(\sigma, A, m)\rangle: \operatorname{tr}(\sigma)=0\},
\end{aligned}
$$

where the last equality follows from the equivalence between traceless matrices and commutators. Now, the quotient space $\mathcal{Q}_{D, m}$ corresponds to $\mathcal{H}_{D, m}^{C P} \cap\left(\mathcal{H}_{D, m}^{M P I}\right)^{\perp}=\mathcal{H}_{D, m}^{C P} \cap \mathcal{H}_{D, m}^{M P S}$. A basis for $\mathcal{Q}_{D, m}$, orthonormal as a subspace of $\left(\mathbb{C}^{d}\right)^{\otimes m}$ can thus be obtained via the following procedure: first, we sequentially generate real random matrices $\sigma^{j}, A_{1}^{j}, \ldots, A_{d}^{j}$, with $\operatorname{tr}\left(\sigma^{j}\right)=0$, which we use to construct a random basis $\left\{\left|\phi_{i}^{[,]}\right\rangle\right\}_{i}$ for $\mathcal{H}_{D, m}^{M P S,[,]}$ (just as we built a basis for $\mathcal{H}_{D, m}^{M P S}$ in Appendix A). Next, we find its orthogonal complement with respect to $\mathcal{H}_{D, m}^{M P S}$, the space of hMPS. This can be done, e.g., by determining the kernel of the $\operatorname{matrix} A_{i j} \equiv\left\langle\phi_{i}^{[,]} \mid \phi_{j}\right\rangle$, where $\left\{\left|\phi_{j}\right\rangle\right\}_{j}$ is a basis for $\mathcal{H}_{D, m}^{M P S}$.

As before, instead of using a randomized algorithm, we can find an orthonormal basis for $\mathcal{H}_{D, m}^{M P S,[,]}$ via polynomial hMPS $\left\{\left|f_{i}\right\rangle\right\}_{i}$ and use it to construct the matrix $\tilde{A}_{i j}=\left\langle f_{i} \mid g_{j}\right\rangle$, where $\left\{\left|g_{j}\right\rangle\right\}_{j}$ denotes an orthonormal basis of polynomials for $\mathcal{H}_{D, m}^{M P S}$. The kernel of $\tilde{A}$ will give us a polynomial basis for $\mathcal{Q}_{D, m}$.

In either case, the dimensionality of all these spaces grows polynomially with the system size $m$, so for small $D$ we can find an orthonormal basis for $\mathcal{H}_{D, m}^{C P}$ for very high values of $m$.

\section{An SDP relaxation for linear opti- mizations over MPS}

Given an $m$-site Hamiltonian $H$, consider the problem 


$$
\begin{array}{ll} 
& \min \operatorname{tr}(H \rho), \\
\text { s.t. } & \rho \in B\left(\mathcal{H}_{D, m}^{M P S}\right), \rho \geq 0, \operatorname{tr}(\rho)=1, \\
& \left(C_{j} \rho C_{j}^{\dagger}\right)^{T_{j}} \geq 0, j=1, \ldots, m-1
\end{array}
$$

where $C_{j}$ is a cut-and-glue operator acting nontrivially over particles $1, \ldots, j$ and $B^{T_{j}}$ denotes the partial transposition of $B$ with respect to the same particle set [19]. Note that we have relaxed the condition of $C_{j} \rho C_{j}^{\dagger}$ being separable to the simpler constraint of being positive under partial transposition. Clearly, the solution of the SDP (19) will provide a lower bound for $\langle H\rangle_{D}$. Let us remark that an implementation of (19) requires explicit bases for $\mathcal{H}_{D, m}^{M P S}$ and $\left\{\mathcal{Q}_{D, j}: j=1, \ldots, m-1\right\}$. These can be obtained efficiently with the non-deterministic algorithms described in Sections A and C.

Suppose now that we are interested in optimizing over TI hMPS given by eq. (3) in the main text. In that case, one can define the following hierarchy of SDPs:

$$
\begin{aligned}
h^{n} \equiv & \min \frac{1}{n-m} \operatorname{tr}\left\{\sum_{i=1}^{n-m} \tau_{i}(H) \rho\right\}, \\
\text { s.t. } & \rho \in B\left(\mathcal{H}_{D, n}^{M P S}\right), \rho \geq 0, \operatorname{tr}(\rho)=1, \\
& \left(C_{j} \rho C_{j}^{\dagger}\right)^{T_{j}} \geq 0, j=1, \ldots, n-1 .
\end{aligned}
$$

Here $\tau$ denotes the translation operator, and is used to enforce TI on the relaxation. Another way to enforce TI is to determine (via., e.g., randomization) the span $\mathcal{S}_{D, n}$ of the $n$-site density matrices of all uniform TI MPS and then demand $\rho \in \mathcal{S}_{D, n}$. In either case, $h^{m} \leq h^{m+1} \leq \ldots \leq h^{\star}$, where $h^{\star}$ is the minimum average value of $H$ over extendible MPS with bond dimension $D$.

For $D=1$, the space $\mathcal{H}_{D, n}^{M P S}$ reduces to the symmetric space of $n d$-dimensional particles, and $C_{j}=\mathbb{I}$ (since all polynomials are central in $D=1$ ). The method hence reduces to the Doherty-Parrilo-Spedalieri (DPS) method for entanglement detection [4]. The DPS method approximates the set of states of the form $\int d \phi p(\phi)|\phi\rangle\left\langle\left.\phi\right|^{\otimes m}\right.$, with $p(\phi) \geq 0$ by partial traces of the set of $n$-symmetric states positive under partial transposition. It can be shown to converge by virtue of the quantum de-Finetti theorem [12]. For $D=\infty$, program (20) is equivalent to computing the $(n-m)^{t h}$ Anderson bound for the Hamiltonian $H$ [2], and convergence can be proven easily. It is an open question whether the hierarchy (20) converges for other values of $D$. 